\title{
EP-169
}

\section{Primary pancreatic Ewing's sarcoma: Dreadful yet treatable alien}

\author{
Adithya MORUMPALLE*, Anand Vijai NATESAN, Palanivelu CHINNUSAMY, Aravinth SUBRAMANIAM \\ Department of HPB \& Minimal Access Surgery, Gem Hospital \& Research Center, Coimbatore, India
}

Introduction: Ewing's sarcoma is a highly aggressive malignant tumour most commonly affecting long bones in children and adolescents. Primary presentation in Pancreas is considered to be an extremely rare and only a handful of cases have been published to date. Our case intensifies the importance to recognize this rare type of tumor in young adults with a primary pancreatic lesion as there is a broad spectrum of tumours with a similar morphological and radiological presentation.

Methods: A 26-year-old man was evaluated for upper abdominal pain with cross sectional imaging studies which revealed $3 \times 3.3 \mathrm{~cm}$ complex cystic lesion in head of pancreas. Serum tumor markers and other blood investigations were within normal limits. We conducted tumour board meeting and proceeded for laparoscopic pancreaticoduodenectomy.

Results: Post-operative whipples specimen was subjected for histopathological analysis and Immunohistochemistry which confirmed the diagnosis of Ewing's sarcoma of pancreas. Currently, the patient is receiving regular follow-up care and received 3 cycles of adjuvant chemotherapy till now and has no evidence of cancer at six months post-surgery.

Conclusions: Primary pancreatic Ewing sarcoma is a very rare highly aggressive malignant tumour that should be considered in differential diagnosis of an unusual pancreatic tumour especially in young adults. Being rare, we only have 32 reported cases up-to-date. Establishing the correct diagnosis requires a multidisciplinary approach involving close cooperation between clinical, radiological and pathological teams with accurate application of immunohistochemistry or cytogenetic analysis. Complete surgical excision with adjuvant chemotherapy and with or without radiation therapy is the standard of care. 